\title{
Impacto de la pandemia COVID-19 en colectivos vulnerables a través de la mirada de los estudiantes de enfermería: Un proyecto educativo con Photovoice
}

\section{Elena Andina Díaz ${ }^{1}$, José Siles González², Néstor Serrano Fuentes ${ }^{3}$, Lindsay Welch ${ }^{3}$, Gema del Blanco Ubiergo ${ }^{1}$ y MCarmen Solano Ruiz ${ }^{2}$}

\author{
${ }^{1}$ Departamento de Enfermería y Fisioterapia, Universidad de León, España | \\ elena.andina@unileon.es; gblau@unileon.es | https://orcid.org/0000-0001-9687-1967; \\ https://orcid.org/0000-0002-9067-9189 \\ 2 Departamento de Enfermería, Universidad de Alicante, España | jose.siles@ua.es; \\ carmen.solano@ua.es | https://orcid.org/0000-0003-3046-639X; https://orcid.org/0000-0001- \\ 8720-8397 \\ ${ }^{3}$ School of Health Sciences, University of Southampton, United Kingdom | n.serrano- \\ fuentes@soton.ac.uk; Iw13e14@soton.ac.uk| https://orcid.org/0000-0003-3085-7593; \\ https://orcid.org/0000-0001-5564-2252
}

\begin{abstract}
Resumen: Introducción: Las nuevas estrategias de enseñanza de los universitarios sugieren la integración de metodologías dinámicas en las que se trabaje activamente su pensamiento crítico. La fotografía reflexiva o Photovoice se ha utilizado en el ámbito educativo con el fin de formar conocimientos sobre la esfera social. Objetivo: desarrollar un proyecto educativo en la que, a través del Photovoice, se promoviera el pensamiento crítico en estudiantes de enfermería. Métodos: Investigación Acción Participativa, concretamente Photovoice. El proyecto educativo se llevó a cabo en tres contextos diferentes: estudiantes de enfermería de la Universidad de León (España), de Alicante (España) y de Southampton (Reino Unido). El tema elegido: el impacto que la pandemia COVID-19 estaba causando en grupos socioeconómicos bajos y minorías étnicas. Los alumnos salieron a la calle y fotografiaron dicho impacto. Cada fotografía la acompañaron de una explicación (narrativa). Posteriormente en el aula se realizó un grupo de discusión, mediante el que analizaron cualitativamente las fotografías/narrativas, estableciendo categorías. Para representar cada categoría, se seleccionaron fotografías. Finalmente se construyó una exposición fotográfica para promover la reflexión en el resto de la comunidad. Resultados: 84 alumnos participaron de proyecto. Las categorías "hambre", "sin hogar", "barrios desfavorecidos", "trabajando en la calle", "silencio en las calles" y "minorías étnicas" que emergieron de los datos sirvieron como hilo para que los estudiantes reflexionaran sobre el impacto que la pandemia COVID-19 estaba causando en grupos socioeconómicos bajos y minorías étnicas. Conclusiones: Este proyecto educativo mostró cómo, a través de la herramienta del Photovoice, se promovió el pensamiento crítico en estos estudiantes, en tres contextos diferentes. El Photovoice parece ser una herramienta original, simple y económica para promover pensamiento crítico de los universitarios respecto a las dimensiones sociales y culturales de la salud. Algo muy importante dado que ellos son futuros promotores de cambios de salud de la comunidad.
\end{abstract}

Palabras clave: Investigación Cualitativa; Photovoice; Estudiantes de Enfermería; Pensamiento Crítico; Colectivos Vulnerables; COVID-19.

Impact of the COVID-19 pandemic on vulnerable groups through the gaze of nursing students: an educational project with Photovoice

\begin{abstract}
Introduction: New teaching strategies for higher education suggest integrating dynamic methodologies. Thus, university students' critical thinking can be actively worked. Reflective photography or photovoice has been used in the educational field to form knowledge about the social context. Goals: To develop an educational project where critical thinking is promoted in nursing students using photovoice. Methods: Participatory Action Research, specifically photovoice. The educational project was carried out in three different geographical areas: nursing students from the University of León (Spain), Alicante (Spain) and Southampton (United Kingdom). The chosen topic was the impact of COVID-19 pandemic on lower socioeconomic groups and ethnic minorities. The students accessed the community context and photographed this impact. Each photograph was accompanied by an explanation (narrative). Then, a discussion group was conducted in seminars where they analysed the photographs/narratives qualitatively, establishing categories. Photographs were selected to represent each category. Finally, a photographic exhibition was built to promote reflection at the community level. Results: 84 students participated in the project. The categories "hunger", "homeless", "disadvantaged neighbourhoods", "working in the streets", "silence in the streets" and "ethnic minorities" emerged from the data and served as a thread for students to reflect on the impact of COVID-19 pandemic on these vulnerable groups. Conclusions: This educational project, which used the photovoice tool, showed how critical thinking was promoted in students across different countries. Photovoice seems to be an original, simple, and inexpensive tool to encourage university students' critical thinking regarding the social and cultural dimensions of health. This is relevant since they are the future promoters of community health changes.
\end{abstract}

Keywords: Qualitative Research; Photovoice; Nursing Students; Critical Thinking; Vulnerable Populations; COVID-19. 


\section{Introducción}

Las nuevas estrategias de enseñanza y aprendizaje de los estudiantes universitarios en ciencias de la salud sugieren la combinación de la educación tradicional, centrada en la adquisición de conocimientos y en la formación de comportamientos, con metodologías en las que se integran dimensiones como los valores, las creencias, los sentimientos, las experiencias o las circunstancias de los estudiantes, así como la creatividad y el pensamiento crítico (Jin \& Bridges, 2016). Estas nuevas tendencias requieren que los estudiantes participen e interactúen directamente en el proceso de aprendizaje, lo que constituye un importante desafío para los académicos. Algunos de los objetivos que estos nuevos enfoques de enseñanza pretenden abordar son los siguientes: adquirir conocimientos, acercar los conceptos teóricos a los prácticos y lograr que los estudiantes lleguen a comprender mejor la naturaleza compleja y/o fenómenos complejos como los relacionados con la salud y el cuidado (Siles-González \& Solano-Ruiz, 2017).

Como docentes, si queremos orientarlos en esa dirección, debemos promover experiencias relacionadas con la salud-enfermedad y el cuidado, además de promover el diálogo crítico. De esta manera, las técnicas y los métodos cualitativos, enmarcados en los paradigmas interpretativos y críticos, se han utilizado de diferentes maneras en el ámbito académico de nuestra disciplina para promover tal reflexión (Edwards et al., 2018; Siles-González \& Solano-Ruiz, 2017).

La fotografía también se ha utilizado en el ámbito educativo reflexivo (Bran et al., 2015; De Lina et al., 2018; Kronk et al., 2015; Mullen et al., 2017), con el fin de crear imágenes y formar conocimientos sobre la esfera social. Así, algunos estudios han utilizado la fotografía participativa o Photovoice. Desde su desarrollo en la década de 1990, a través de una investigación centrada en contextos de conciencia crítica y teoría feminista, el método se ha estado utilizando en la educación sanitaria y en campos conexos (Díez et al., 2017; Evans-Agnew et al., 2017; García Leal et al., 2018; Johnston, 2016).

Encontramos escasos estudios en los que el Photovoice haya sido utilizado como herramienta educativa en estudiantes universitarios de diferentes disciplinas, entre ellas, de ciencias de la salud (Andina-Díaz, 2020; Coronado et al., 2020; Fernandes et al., 2018; Gallagher \& Stevens, 2015). Por ello, nos planteamos la siguiente pregunta de investigación: ¿la utilización del photovoice en estudiantes universitarios favorece el pensamiento crítico? El hecho de realizar investigaciones utilizando dicha herramienta, en diferentes contextos, nos ayudará a ampliar el conocimiento sobre su utilidad.

El objetivo de este trabajo se centró en desarrollar un proyecto educativo en estudiantes de enfermería el que, a través de la herramienta del Photovoice, se promoviera el pensamiento crítico y la reflexión con respecto a la afectación de la pandemia en grupos vulnerables.

\section{Metodología}

\subsection{Diseño}

Investigación Acción Participativa (IAP), concretamente Photovoice. El Photovoice es una metodología/herramienta enmarcada dentro de la IAP que permite a las personas "identificar, representar y mejorar su comunidad a través de una técnica fotográfica específica" (Wang \& Burris, 1997). Siguiendo sus pautas básicas, en nuestro estudio, esta fotografía participativa nos permitió: i) que los estudiantes registraran y reflejaran las fortalezas y las preocupaciones de un tema, y ii) se promoviera el diálogo crítico y el conocimiento sobre cuestiones importantes mediante el debate de las fotografías en grupos pequeños y grandes. 


\subsection{Emplazamiento y Participantes}

Este proyecto se llevó a cabo en tres contextos diferentes por separado. Por una parte, en la Universidad de León (España), con estudiantes matriculados en la asignatura "Proyectos de Cooperación Sanitaria y Ayuda Humanitaria" (4 curso, Grado Enfermería, campus Vegazana). Por otra parte, en la Universidad de Alicante (España), con estudiantes matriculados en la asignatura "Cultura de los Cuidados, Educación para el Desarrollo y Pensamiento Crítico" (4 curso, Grado Enfermería). Por otra parte, en la Universidad de Southampton (Reino Unido), con estudiantes matriculados en la asignatura "Global and Public Health" ( $1^{\circ}$ curso, Enfermería).

Se invitó a todos los estudiantes matriculados en las tres asignaturas que participaran de manera voluntaria.

\subsection{Procedimiento}

Este proyecto educativo estuvo compuesto por 3 sesiones. Las 3 sesiones se llevaron a cabo durante los meses de noviembre/diciembre de 2020 con los estudiantes de la Universidad de Alicante, durante los meses de enero/febrero de 2021 con los estudiantes de la Universidad de León, y durante los meses de febrero-marzo de 2021 con los estudiantes de la Universidad de Southampton.

El tema con el que se trabajó fue el impacto que la pandemia COVID-19 estaba causando en grupos socioeconómicos bajos y minorías étnicas. Un tema que estaba alineado con los contenidos docentes de las tres asignaturas.

En una primera sesión (60 min), en el aula:

En primer lugar, se explicaron las características básicas de los colectivos vulnerables en los que debían centrar su mirada, grupos socioeconómicos bajos y minorías étnicas, incidiendo en cómo la COVID-19 estaba impactando en sus vidas, por acrecentar las disparidades sociales, económicas, o relativas a la salud, entre otras (United Nations, 2020), con varios ejemplos.

Seguidamente se expusieron los conceptos básicos de la metodología del Photovoice, incidiendo en 3 pasos: Fotodocumentación, Fotoelicitación y Exposición en Galería (Wang \& Burris, 1997).

Se les explicó lo que debían realiza en la siguiente sesión: salir a la calle y, ayudados con sus móviles/tablets/cámaras, tomar 3 fotografías en su comunidad centradas en el objetivo expuesto: "retratar el impacto que la COVID-19 ha causado en la vida/salud de colectivos vulnerables (minorías étnicas y grupos socioeconómicos bajos)". Cada una de las 3 fotografías debía ser acompañada por una narrativa, siguiendo el modelo SHOWED, específico del Photovoice (Wallerstein \& Bernstein, 1988; Wang \& Burris, 1997). Dicho modelo cuenta con 6 preguntas a las que hay que dar respuesta: ¿Qué se ve en esta fotografía?, ¿Qué está realmente pasando?, ¿Cómo se relaciona esto con nuestras vidas?, ¿Por qué existe este problema?, ¿Cómo puede esta imagen educar a la gente?, ¿Qué podemos hacer al respecto? Para ello se les entregaron copias de dicho modelo.

Se les entregó también copias de los Consentimientos Informados pertinentes (Aspectos éticos).

En una segunda sesión (120 min), se realizó la salida a la comunidad (Fotodocumentación). Cada alumno, solo o en grupo, eligió la zona/barrio de la ciudad en la que sacar las 3 fotografías, así como las escenas/personajes. 
En una tercera sesión (120 min), en el aula, se llevó a cabo una exposición de las fotografías/narrativas realizadas por los alumnos y un grupo de discusión para reflexionar sobre ellas. Inicialmente se distribuyeron a todos los alumnos de la asignatura en dos grupos (dos clases) de 20-25 alumnos.

Dentro del aula, se formaron grupos de 5 estudiantes (4-5 grupos por aula). En primer lugar, en cada grupo de 5, cada estudiante debía explicar a los otros 4 las tres fotografías seleccionadas utilizando sus narraciones basadas en SHOWED. Se generó un debate dentro del grupo, en el que seleccionaron las cinco fotografías/narrativas SHOWED que reflejaban mejor la realidad de esos colectivos.

En segundo lugar, se formó un debate entre todos los estudiantes del aula. Con todas las fotografías/narrativas SHOWED (5 por grupo, 20-25 por aula), los estudiantes realizaron un análisis de estas, estableciendo diferentes temas o categorías. Para representar cada una de esas categorías, se seleccionaron algunas fotografías (Fotoelicitación).

Finalmente, las fotografías se expusieron en las paredes de la facultad (simulando una Exposición en galería), con el fin de promover la reflexión entre el resto de la comunidad universitaria.

\subsection{Análisis de Datos}

Se realizó un análisis de contenido temático de las fotografías/narrativas, de manera grupal, en la tercera sesión. En este análisis participaron todos los estudiantes, estableciendo categorías. Los profesores dieron pautas y guiaron a los estudiantes en dicho proceso.

Posteriormente, dos miembros del equipo investigador realizaron un análisis de contenido temático conjunto, con los datos de los tres contextos, reagrupando las categorías y validando. Para ello, se siguieron los pasos propuestos por Giorgi (1997): (i) lectura detallada de las narrativas, así como una observación de todas las fotografías; (ii) extracción de código y agrupación en categorías; (iii) el contenido de cada una de las categorías analizadas fue interpretado considerando las reflexiones descritas en las narrativas y las categorías que habían surgido en los grupos de debate en el aula. No se utilizó ningún marco predeterminado para realizar el análisis de datos cualitativos. Los códigos y categorías se extrajeron de las narrativas y las fotografías. Se utilizó el software MAXQDA $2020 \AA$ para respaldar este análisis. Los resultados fueron mostrados al resto de miembros del equipo de investigación, para llegar a un consenso, manteniendo en todo momento una actitud reflexiva y crítica.

\subsection{Aspectos Éticos}

Los estudiantes firmaron un Consentimiento Informado (voluntariedad, cesión de imágenes, confidencialidad). Los estudiantes llevaron una copia de un Consentimiento Informado para dárselo a firmar a las personas que aparecieran en sus fotografías (voluntariedad, cesión de imágenes, confidencialidad). Este proyecto cuenta con el informe favorable de los Comités de Ética de las tres universidades implicadas (ETICA-ULE-0392020; UA-2020-12-04; US-62375). 
Vol. 8 | Investigación Cualitativa en Salud: Avances y Desafíos

\section{Resultados}

En el proyecto educativo participaron 84 estudiantes de las tres universidades.

De las fotografías y narrativas surgieron 6 categorías: "hambre", "sin hogar", "barrios desfavorecidos", "trabajando en la calle", "silencio en las calles", "minorías étnicas". Todas ellas sirvieron como hilo para que los estudiantes reflexionaran sobre el impacto que la pandemia COVID-19 estaba causando en grupos socioeconómicos bajos y minorías étnicas.

\subsection{Hambre}

En esta categoría los estudiantes incluyeron historias en las que se hablaba del hambre. Se reflexionó sobre cómo a causa de la pandemia, muchas familias se habían quedado sin trabajo, por tener que estar confinados y/o por quiebra de negocios al no ser rentables ya. Este hecho trajo como consecuencia que no contaran con recursos económicos suficientes para poder satisfacer sus necesidades básicas, entre ellas, la de alimentación.

En las figuras 1, 2 y 3 se muestran imágenes de bolsas de alimentos, sobre una mesa de un banco de alimentos, la entrada de una Organización No Gubernamental donde reparten alimentos a gente con bajos recursos, y un hombre buscando comida en un contenedor.

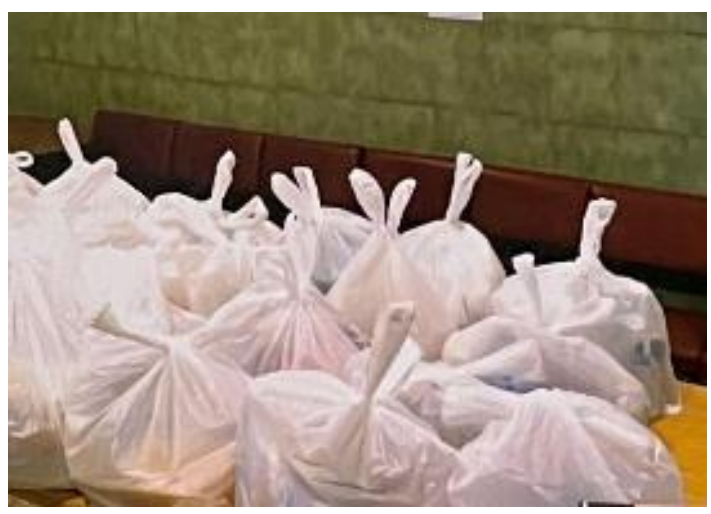

Fig. 1. Buscando ayuda de la caridad.

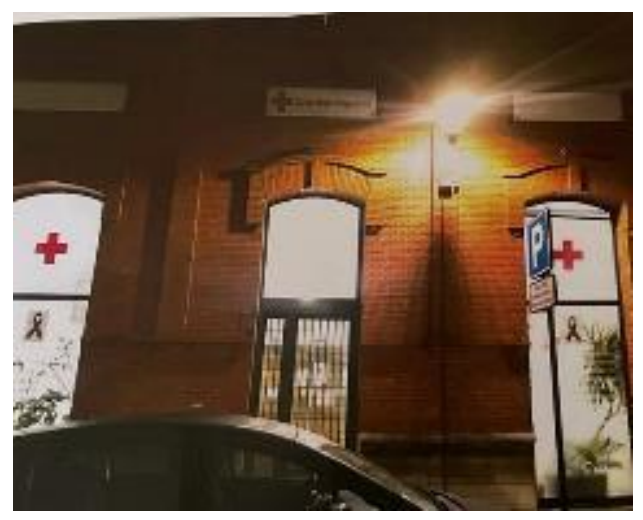

Fig. 2. Cruz Roja.

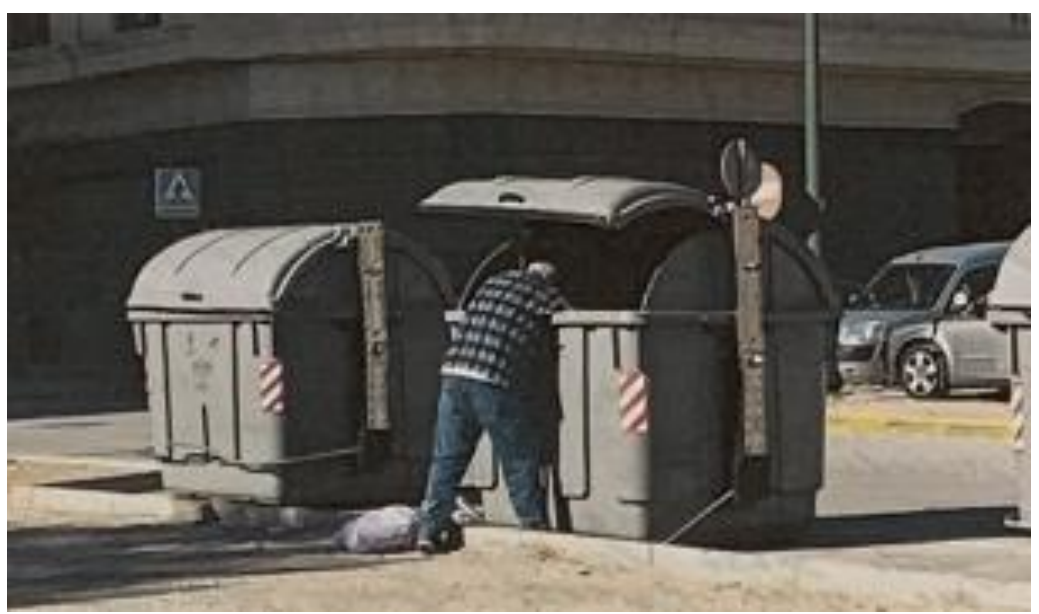

Fig. 3. Buscando en contenedores. 
Vol. 8 | Investigación Cualitativa en Salud: Avances y Desafíos

\subsection{Sin Hogar}

En esta categoría los estudiantes incluyeron ilustraciones en las que se abordaba el tema de las personas sin hogar, y cómo sus vidas se habían visto seriamente afectadas por las medidas impuestas a consecuencia de la pandemia (uso de mascarilla, higiene extrema, distancia de seguridad...). En las figuras 4, 5 y 6 se retrató el día a día de algunas personas sin hogar.

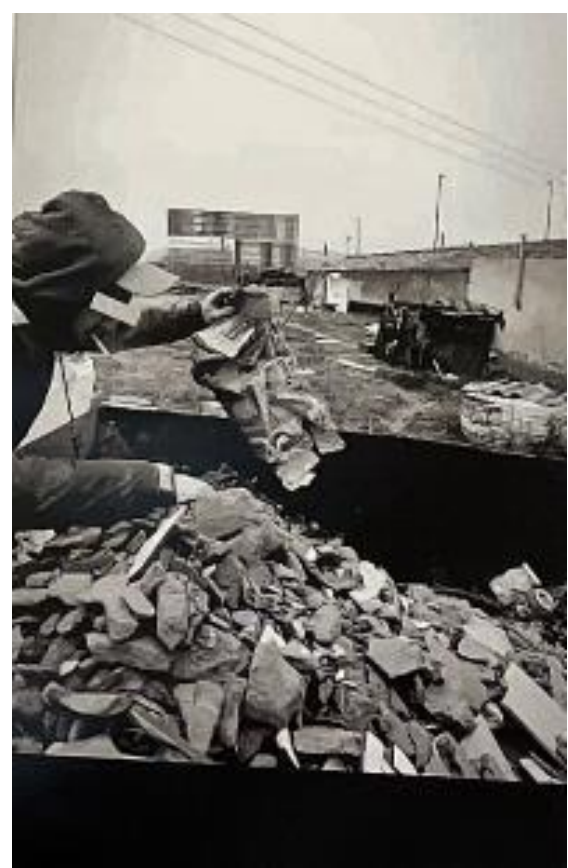

Fig. 4. Cazarrecompensas.

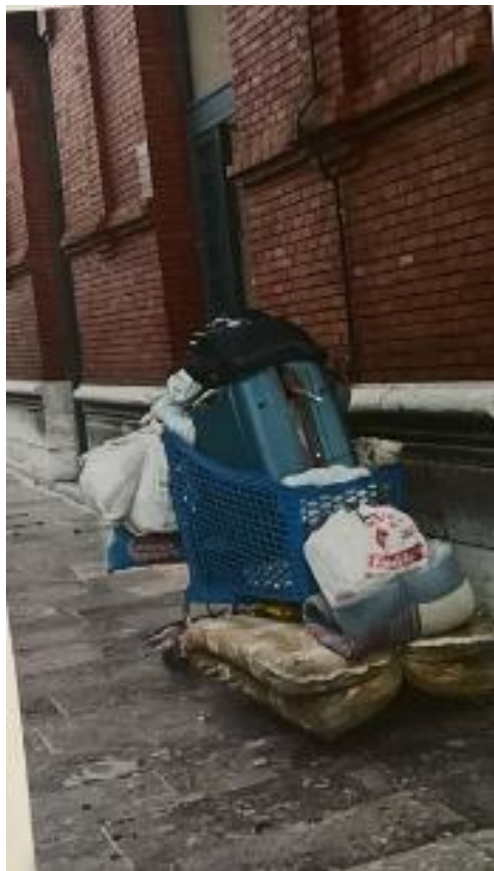

Fig. 5. Mi casa en pandemia.

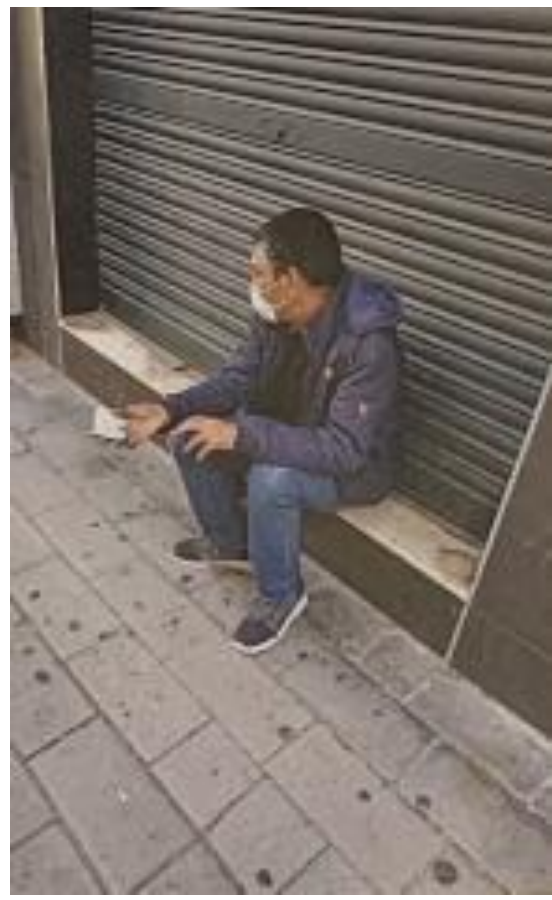

Fig. 6. Vendo pañuelos. 
Vol. 8 | Investigación Cualitativa en Salud: Avances y Desafíos

\subsection{Barrios Desfavorecidos}

En esta categoría los estudiantes mostraron algunos barrios desfavorecidos de su ciudad, como se ve en las figuras 7,8 y 9 .

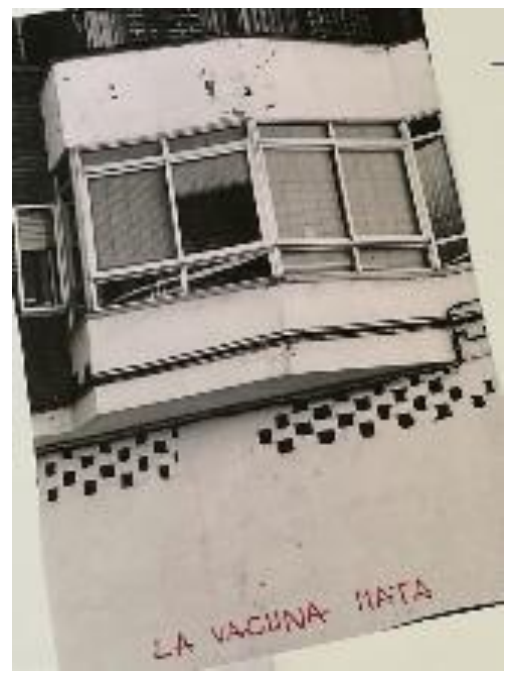

Fig. 7. Cultura y Covid.

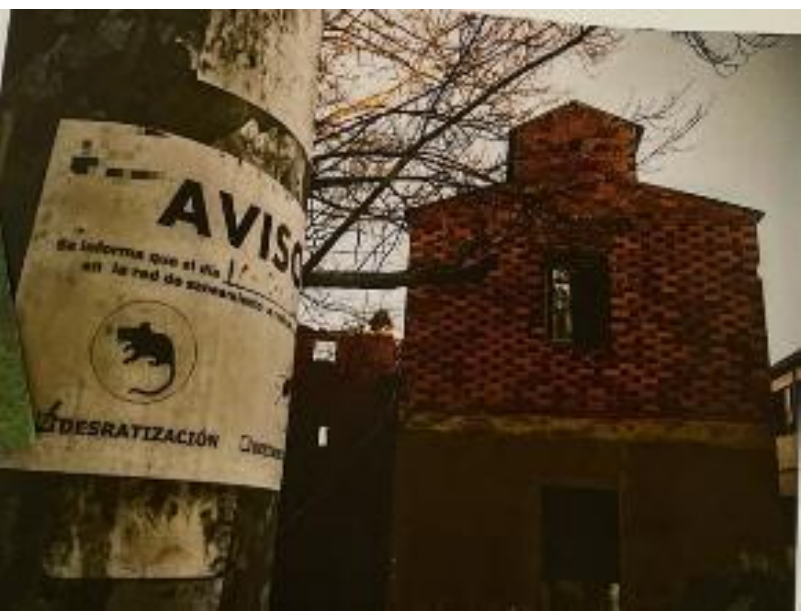

Fig. 8. Mal barrio.

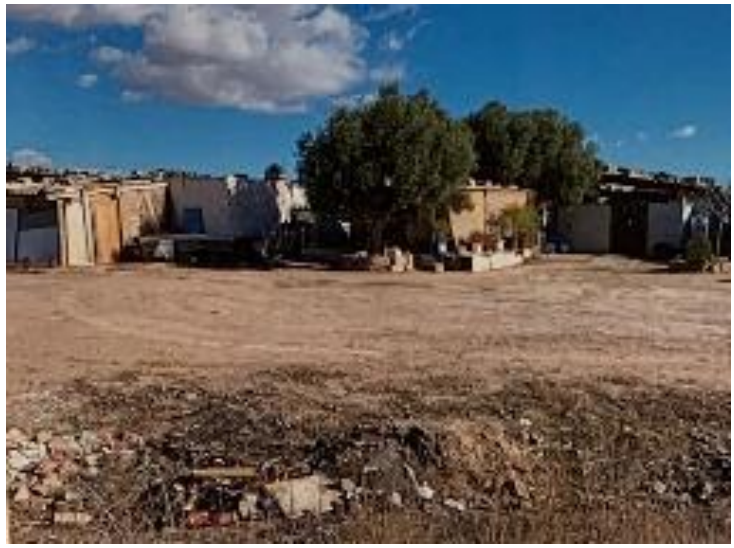

Fig. 9. Búsqueda. 
Vol. 8 | Investigación Cualitativa en Salud: Avances y Desafíos

\subsection{Trabajando en la Calle}

En esta categoría los estudiantes incluyeron historias de personas que contaban con diferentes trabajos/oficios que, antes de la pandemia, se desarrollaban en la calle, y que como consecuencia de la pandemia tuvieron que readaptarse o cerrar. Ejemplos de ello fueron el ocio ambulante (figura 10), o los mercados ambulantes (figuras 11 y 12).

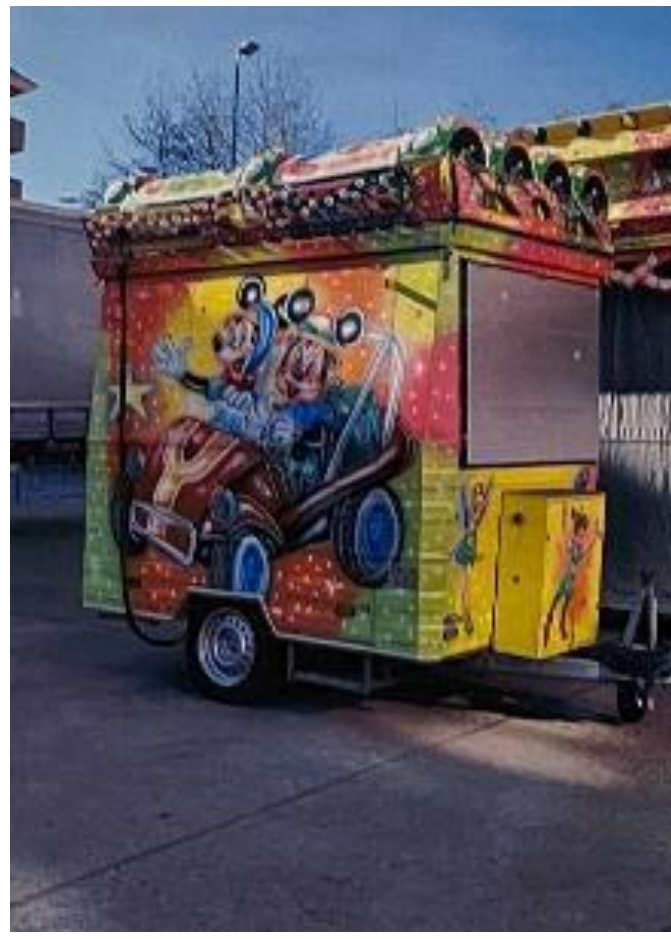

Fig. 10. Ocio ambulante.

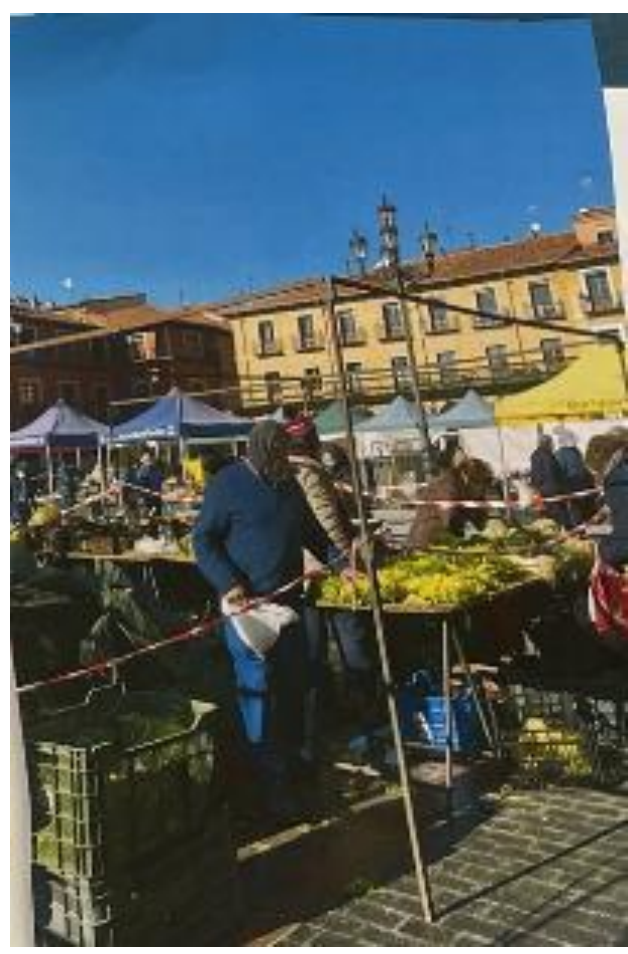

Fig. 11. Mercado.

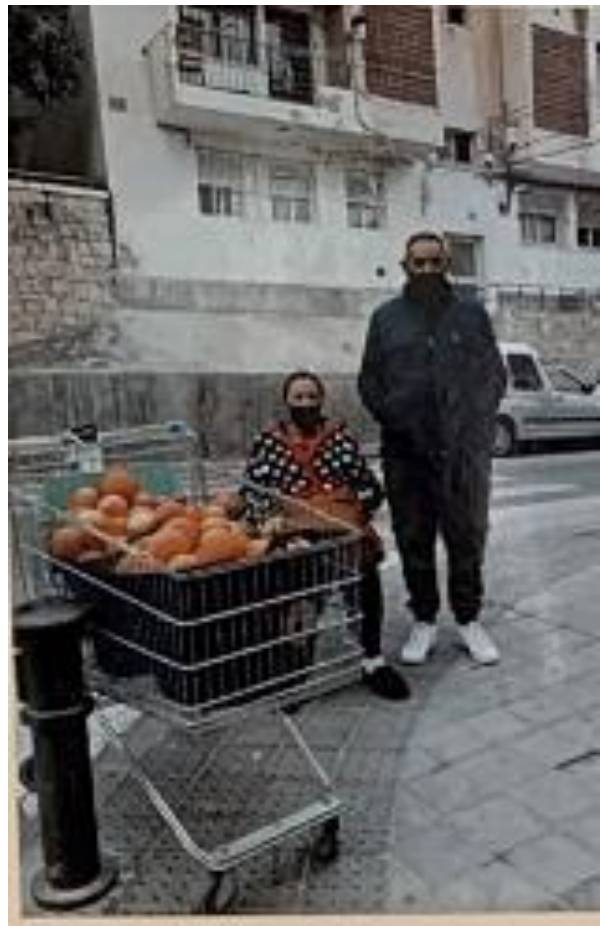

Fig. 12. No hay mercado. 


\subsection{Silencio de Calles}

En esta categoría los estudiantes discutieron el impacto que la pandemia estaba teniendo en los autónomos y pequeños comercios, como tiendas y bares. Sus cierres obligados estaban afectando a sus vidas a nivel económico y de salud (figuras 13 y 14).

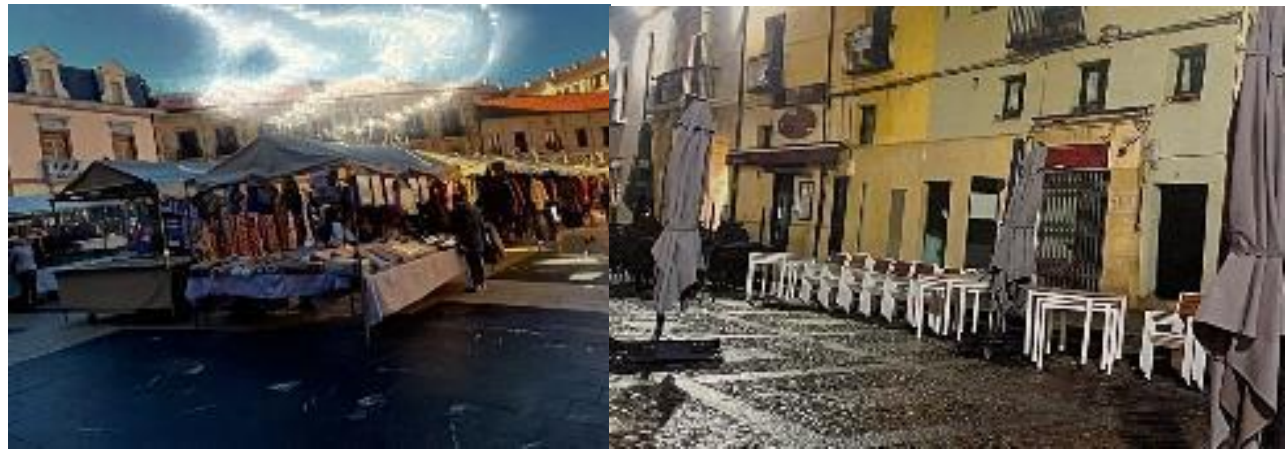

Fig. 13. Cierre obligado.

Fig. 14. La otra cara del covid.

\subsection{Minorías Étnicas}

En esta categoría los estudiantes hablaron del impacto de la pandemia en los colectivos de inmigrantes, resaltando la necesidad de crear lazos, y extenderlos al resto de la comunidad, para sobrevivir (figuras 15 y 16).

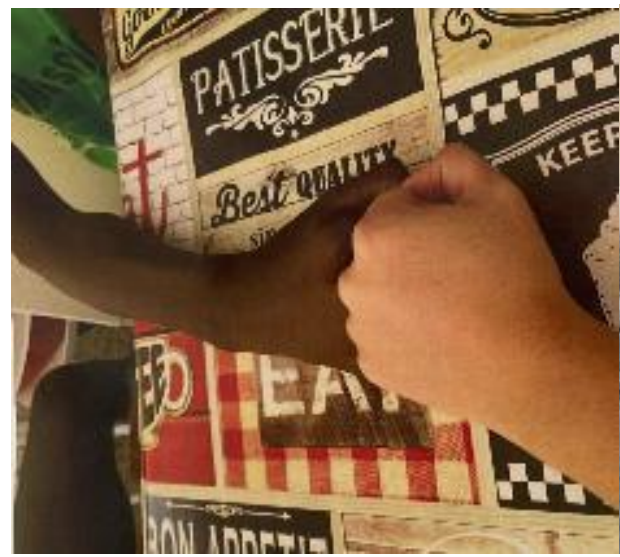

Fig. 15. Unión.

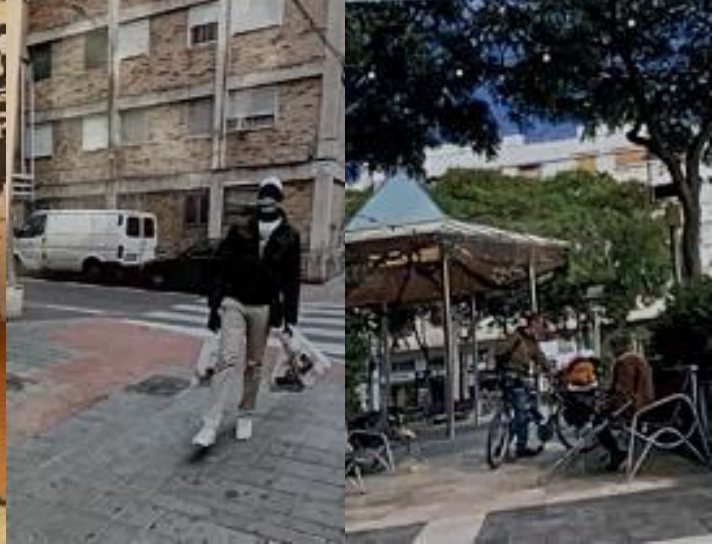

Fig. 16. Lazos entre iguales.

\section{Discusión y Conclusiones}

Este proyecto educativo describe cómo, a través de la herramienta del Photovoice, se consiguió promover el pensamiento crítico en estos grupos de estudiantes, en tres contextos diferentes. Los alumnos salieron a la calle para fotografiar el impacto que la pandemia COVID-19 había originado en grupos socioeconómicos bajos y minorías étnicas en su ciudad. Para ello tuvieron que pensar previamente en los lugares/situaciones/colectivos afectados a visitar, y en cómo retratarlos. Posteriormente, el debate establecido en el aula, exponiendo las fotografías y narrativas, y el análisis/categorización, sirvió para estimular su pensamiento crítico al respecto.

En este caso, el Photovoice tuvo un efecto pedagógico significativo, ayudando a que los estudiantes de enfermería empatizaran y conocieran las dimensiones sociales y culturales de la salud, en un contexto de pandemia, a través de la reflexión y el pensamiento crítico. Algo muy importante, dado que ellos son futuros promotores de cambios de salud de la comunidad. 
No son muchos los estudios que se han encontrado publicados en los últimos años en los que se haya trabajado con el Photovoice como herramienta pedagógica en el ámbito de ciencias de la salud (Andina-Díaz, 2020; Coronado et al., 2020; Fernandes et al., 2018; Gallagher \& Stevens, 2015), así como en otras disciplinas (Malka, 2021; Von Sommoggy et al., 2020). Los resultados de este estudio ayudan a dar consistencia al Photovoice como herramienta válida para tal fin. Además, la presentan como una técnica original, simple y económica.

Este trabajo cuenta con algunas limitaciones, como la homogeneidad de la muestra (el estudio se realizó sólo con estudiantes de enfermería), así como la forma en la que se recogieron las impresiones de los estudiantes (debate en el aula). Por ello, como futuras líneas de investigación sería interesante ponerla en práctica con otras temáticas, así como en estudiantes de otras titulaciones. Por último, sería pertinente recoger la opinión de los estudiantes paralelamente a través otras herramientas (cuestionarios, por ejemplo).

\section{Referencias}

Andina-Díaz, E. (2020). Using Photovoice to stimulate critical thinking: An exploratory study with Nursing students. Revista Latino-Americana Enfermagem, 28:e3314. http://dx.doi.org/10.1590/1518-8345.3625.3314

Bran, G., Miller, K., Saunde, R., Dugmore, H., Etherton-Beer, C. (2015). Expanding the Caring Lens: Nursing and Medical Students Reflecting on Images of Older People. Gerontology \& Geriatric Education, 37(2). https://doi.org/10.1080/02701960.2015.1059832

Coronado, C., Freijomil-Vázquez, C., Fernández-Basanta, S., Andina-Díaz, E., Movilla-Fernández, M. J. (2020). Using photovoice to explore the impact on a student community after including cross-sectional content on environmental sustainability in a university subject: a case study. International Journal of Sustainability in Higher Education, 21(7), 1331-1350. https://doi.org/10.1108/lJSHE-01-2020-0031

De Lima, R., Bergold, L.B., Souza, J.D.F., Barbosa, G.S., Ferreira, M.A. (2018). Death education: sensibility for caregiving. Revista Brasileira Enfermagem, 71(4), 1779-84. https://doi.org/10.1590/0034-7167-2017-0018

Díez, J., Conde, P., Sandin, M., Urtasun, M., López, R., Carrero, J.L., et al. (2017). Understanding the local food environment: A participatory photovoice project in a low-income area in Madrid, Spain. Health Place, 43, 95-103. https://doi.org/10.1016/j.healthplace.2016.11.012

Edwards, S., Fryer, N., Boot, M., Farquharson, M., McCormarck, S., Sluman, K., Tigar, K. (2018). Results of cross-faculty 'capstone' assessments involving nursing and performing arts students. Nursing Management, 25(4), 22-9. https://doi.org/10.7748/nm.2018.e1777

Evans-Agnew, R.A., Boutain, D.M., Rosemberg, M.A. (2017). Advancing nursing research in the visual era. Reenvisioning the Photovoice Process Across Phenomenological, Grounded Theory, and Critical Theory Methodologies. ANS Advances in Nursing Science, 40(1), E1-15. https://doi.org/10.1097/ANS.0000000000000159

Fernandes, C.S., Ferreira, F., Marques, G. (2018). The use of the Photovoice methodology to determine the concept of family which nursing students have. Avances en Enfermería, 36(1), 59-68. https://doi.org/10.15446/av.enferm.v36n1.63988

Gallagher, M.R., Stevens, C.A. (2015). Adapting and Integrating Photovoice in a Baccalaureate Community Course to Enhance Clinical Experiential Learning. Journal of Nursing Education, 54(11), 659-62. https://doi.org/10.3928/01484834- 20151016-09

Garcia Leal, C.C., Gomes-Sponholz, F.A., Mamede, F.V., Lossi-Silva, M.A., Baptista-Oliveira, N.T., Moraes-Leite, A. (2018). Photovoice: method experiment research with adolescent mothers. Escola Anna Nery, 22(3), e20170322. https://doi.org/10.1590/2177-9465-ean-2017-0322

Giorgi, A. (1997). The theory, practice, and evaluation of the phenomenological method as a qualitative research procedure. Journal of Phenomenological Psychology, 28(2), 235-260. https://doi.org/10.1163/156916297X00103 
Vol. 8 | Investigación Cualitativa en Salud: Avances y Desafíos

Jin, J., Bridges, S. (2016). Qualitative Research in PBL in Health Sciences Education: A Review. Interdisciplinary Journal of Problem-Based Learning, 10(2). https://doi.org/10.7771/15415015.1605

Johnston, G. (2016). Champions for social change: photovoice ethics in practice and false hopes for policy and social change. Global Public Health, 11(5-6), 799-811. https://doi.org/10.1080/17441692.2016.1170176

Kronk, R., Weideman, Y., Cunningham, L., Resick, L. (2015). Capturing students transformation from a global service-learning experience: the efficacy of photo-elicitation as a qualitative research method. Journal of Nursing Education, 54(9), S99-S102. https://doi.org/10.3928/01484834- 20150814-18

Malka, M. (2021). Photovoice as a creative coping tool with the COVID-19 crisis in practical training seminar for social work students. Qualitative Social Work, 20(1-2), 544-552. https://doi.org/10.1177/1473325020973309

Mullen, R.F., Kydd, A., Fleming, A., McMillan, L. (2017). Dignity in nursing care: what does it mean to nursing students? Nursing Ethics, 1:969733017720825. https://doi.org/10.1177/0969733017720825

Siles-Gon'zalez, J., Solano-Ruiz, C. (2017). Poesía y cuidados: un instrumento para la gestión de emociones y sentimientos en enfermería. Enfermería: Cuidados Humanizados, 6(2), 23936606. https://doi.org/10.22235/ech.v6i2.1463

United Nations. (2020). UN working to ensure vulnerable groups not left behind in COVID-19 response. Available at: https://www.un.org/en/un-coronavirus-communications-team/unworking-ensure-vulnerable-groups-not-left-behind-covid-19

Von Sommoggy, J., Rueter, J., Curbach, J., Helten, J., Tittlbach, S., \& Loss, J. (2020). How Does the Campus Environment Influence Everyday Physical Activity? A Photovoice Study Among Students of Two German Universities. Frontiers in public health, 8, 561175. https://doi.org/10.3389/fpubh.2020.561175

Wallerstein, N., Bernstein, E. (1988). Empowerment education: Freire's ideas adapted to health education. Health Education Behaviour, 15, 379-394.

Wang, C.C., Burris, M.A.(1997). Photovoice: Concept, Methodology and Use for Participatory needs Assessment. Health Education Behaviour, 24(3), 369-87. 